\title{
A robust design for accessible text entry
}

\author{
Jacob O. Wobbrock \\ Human-Computer Interaction Institute \\ School of Computer Science \\ Carnegie Mellon University \\ Pittsburgh, PA 15213 \\ E-mail: jrock@cs.cmu.edu \\ http://www.edgewrite.com/
}

\begin{abstract}
This paper describes the author's dissertation research on designing, implementing, and evaluating the EdgeWrite text entry method. The goal of this research is to develop a method that is highly "robust," remaining accessible and accurate across a variety of devices, abilities, circumstances, and constraints. EdgeWrite is particularly aimed at users with motor impairments and able-bodied users "on the go." To date, this research has resulted in versions of EdgeWrite for PDAs, touchpads, displacement joysticks, isometric joysticks, trackballs, 4-keys, and more, all of which use the same EdgeWrite alphabet and concepts. The stylus version, for instance, has been shown to be significantly more accurate than Graffiti for both able-bodied and motor-impaired users. Similarly, the trackball version has been shown to be better than on-screen keyboards for some people who use trackballs due to motor impairments. This paper discusses these and other achievements, and points towards future work on a mobile phone version for situationally-impaired users. From its inception, EdgeWrite has been developed with the help of participants, both able-bodied and motor-impaired.
\end{abstract}

\section{Introduction}

The entry of text places significant physical demands on users with motor impairments. Tremor, spasm, poor coordination, low strength, and rapid fatigue make it difficult to enter text with a QWERTY keyboard or PDA stylus. Impaired users have particular difficulty entering text on small devices that require controlled movements [4]. And as small devices proliferate in an aging population, the number of users with motor impairments will only increase. Although work exists on accessible text entry for desktops, little work exists on accessible text entry for handhelds. The "digital divide" threatens to grow if handheld devices are not made more accessible.

But motor-impaired users are not the only people who need accessible devices. Ablebodied users incur "situational impairments" [5] when using mobile devices "on the go." The spatial constraints of small devices necessitate the use of compact (if not cramped) input techniques demanding accurate movement and careful control. These demands are intensified while walking or riding, since use in motion can reduce visibility, divide attention, and cause physical instability through vibrational tremor. But unfortunately, most mobile text entry methods are not sufficiently tactile or physically stable to be used accurately while in motion. 


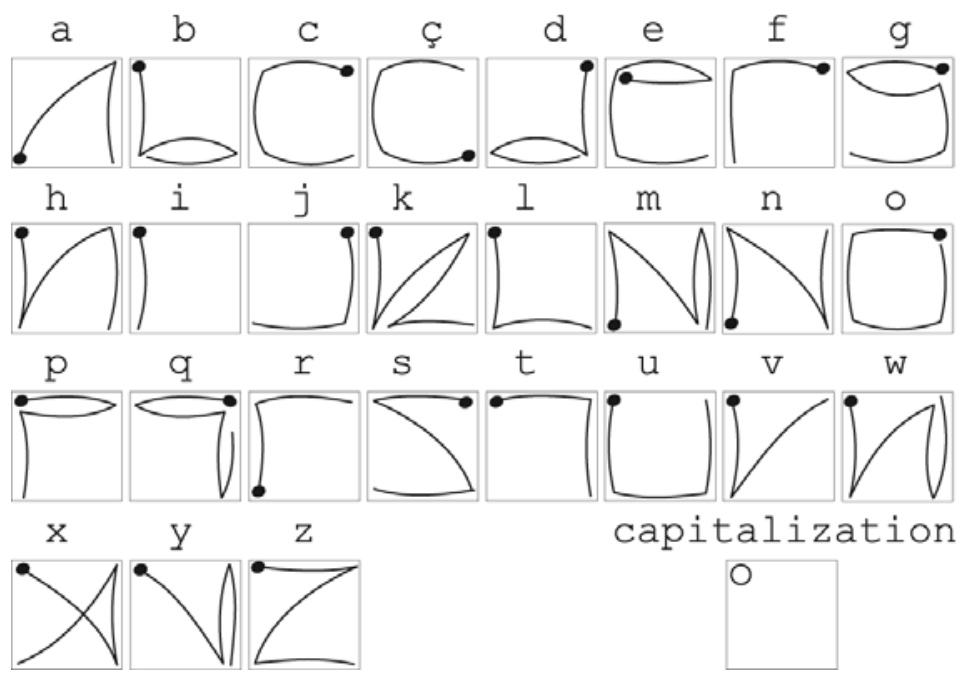

Figure 1. The EdgeWrite alphabet, which is the same for all EdgeWrite devices, was designed by participants to maximize guessability [7]. Multiple versions of each letter exist to increase guessability (not shown). A full character chart can be found at http://www.edgewrite.com.

Thus, the need for physical stability, tactility, accuracy, and control which is exists in motor-impaired text entry also exists in mobile text entry. Accordingly, a design successful in one domain may also be successful in the other. To this end, I have been developing a "robust" design for text entry called EdgeWrite (Figure 1). Its core concept is to use physical edges and corners to provide motor stability for good accuracy despite physical demands. EdgeWrite's robustness takes these forms:

- Multi-device adaptability: EdgeWrite can be easily instantiated on a variety of devices, allowing users with motor impairments to switch among devices to distribute strain and fatigue. This property also allows EdgeWrite to work on new handheld devices, which continually emerge and are ever-smaller.

- High compact-ibility: EdgeWrite remains effective even in very small spaces. For example, our studies show the stylus version can work in a square as small as 7 $\mathrm{mm}$ on a side. Our isometric joystick version is also highly compact. Compactibility is important for small devices and for reducing the distance people must move.

- Recognition despite tremor: Recognition is robust even in the face of wiggle or tremor, since only the order in which the four corners are hit is used to recognize letters, not the overall path of motion [10].

- Situational factors: EdgeWrite is robust in the face of situational factors such as divided attention and the vibrational tremor caused by walking or riding. EdgeWrite's tactile edges provide passive haptic feedback and physical stability, which aid in compromised text entry situations. 


\section{Key goals and results}

The main goals of this research are:

(1) demonstrating robustness and versatility for both motor-impaired and situationally-impaired users,

(2) creating useful real-world input techniques for these groups of users, and

(3) contributing fundamental advancements to the methods of text entry design and evaluation.

Although there has been work on multi-device text entry (e.g. [1]), and much recent work on text entry method development [3], none has been devoted to overcoming impairments. Instead, most is focused on high-performance handheld entry for ablebodied users (e.g. [2]).

Specifically, I wish to show EdgeWrite's effectiveness on three devices for three separate contexts (Figure 2):

(1) On a PDA with a stylus for people with tremor,

(2) on a trackball for motor-impaired trackball users, and

(3) on a mobile phone for able-bodied users who are walking.

These three contexts were chosen to highlight the breadth of EdgeWrite designs. Of them, the first has had the most progress, while the second is under development and the third is in design.

To date, we have shown the stylus version of EdgeWrite to be over 18\% more accurate than Graffiti for able-bodied users and 2-4 times more accurate for some users with motor impairments [10]. We have also implemented EdgeWrite for power wheelchair joysticks and touchpads as integrated control devices for desktop text entry [9]. Our trackball version was significantly faster than a trackball-controlled on-screen keyboard over multiple sessions for a user with spinal cord injury [8]. A former user of on-screen keyboards for 15 years, he now uses Trackball EdgeWrite instead.

In addition, we have developed methods of improving the guessability of symbolic alphabets [7] and in creating algorithms for improving text entry error rate analysis by extending the work of Soukoreff and MacKenzie [6] on text entry method evaluation.
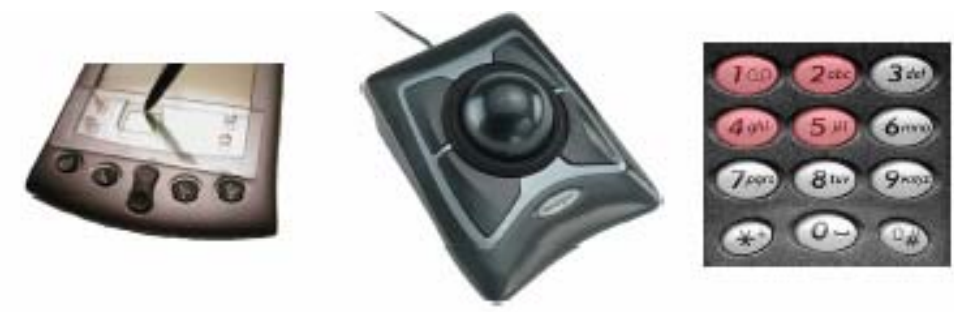

Figure 2. EdgeWrite will be designed, implemented, and evaluated for use on a PDA with a stylus by people with tremor, on a trackball by motor-impaired trackball users, and on a mobile phone by able-bodied users who are walking or riding. 


\section{References}

1. Isokoski, P. and Raisamo, R. (2000) Device independent text input: A rationale and an example. Proc. AVI 2000. New York: ACM Press, 76-83.

2. Kristensson, P. and Zhai, S. (2004) SHARK2: A large vocabulary shorthand writing system for pen-based computers. Proc. UIST 2004. New York: ACM Press, 43-52.

3. MacKenzie, I.S. and Soukoreff, R.W. (2002) Text entry for mobile computing: Models and methods, theory and practice. Human Computer Interaction 17 (2), 147-198.

4. Myers, B.A., Wobbrock, J.O., Yang, S., Yeung, B., Nichols, J. and Miller, R. (2002) Using handhelds to help people with motor impairments. Proc. ASSETS 2002. New York: ACM Press, 89-96.

5. Sears, A., Lin, M., Jacko, J. and Xiao, Y. (2003) When computers fade: Pervasive computing and situationally induced impairments and disabilities. Proc. HCI Int'l 2003, vol. 2. Elsevier Science, 1298-1302.

6. Soukoreff, R.W. and MacKenzie, I.S. (2003) Metrics for text entry research: An evaluation of MSD and KSPC, and a new unified error metric. Proc. CHI 2003. New York: ACM Press, 113-120.

7. Wobbrock, J.O., Aung, H.H., Rothrock, B. and Myers, B.A. (2005) Maximizing the guessability of symbolic input. Extended Abstracts CHI 2005. New York: ACM Press, 18691872.

8. Wobbrock, J.O. and Myers, B.A. (2006) Trackball text entry for people with motor impairments. Proc. CHI 2006. New York: ACM Press, in press.

9. Wobbrock, J.O., Myers, B.A., Aung, H.H. and LoPresti, E.F. (2004) Text entry from power wheelchairs: EdgeWrite for joysticks and touchpads. Proc. ASSETS 2004. New York: ACM Press, 110-117.

10. Wobbrock, J.O., Myers, B.A. and Kembel, J.A. (2003) EdgeWrite: A stylus-based text entry method designed for high accuracy and stability of motion. Proc. of UIST 2003. New York: ACM Press, 61-70. 